\title{
Bidirectional Relations Between Parenting and Prosocial Behavior for Asian and European-American Emerging Adults
}

\author{
Laura M. Padilla-Walker \\ Brigham Young University - Provo \\ Larry J. Nelson \\ Brigham Young University - Provo, larry_nelson@byu.edu \\ Xinyuan Fu \\ Central University of Finance and Economics \\ Carolyn McNamara Barry \\ Loyola University Baltimore \\ Follow this and additional works at: https://scholarsarchive.byu.edu/facpub \\ Part of the Other Social and Behavioral Sciences Commons
}

\section{Original Publication Citation}

Padilla-Walker, L. M., Nelson, L. J., Barry, C. M., \& *Fu, X. (2018). Bidirectional relations between parenting and prosocial behavior for Asian and European American emerging adults. Journal of Adult Development, 25, 107-120.

\section{BYU ScholarsArchive Citation}

Padilla-Walker, Laura M.; Nelson, Larry J.; Fu, Xinyuan; and Barry, Carolyn McNamara, "Bidirectional Relations Between Parenting and Prosocial Behavior for Asian and European-American Emerging Adults" (2017). Faculty Publications. 4703.

https://scholarsarchive.byu.edu/facpub/4703

This Peer-Reviewed Article is brought to you for free and open access by BYU ScholarsArchive. It has been accepted for inclusion in Faculty Publications by an authorized administrator of BYU ScholarsArchive. For more information, please contact ellen_amatangelo@byu.edu. 


\title{
Bidirectional Relations Between Parenting and Prosocial Behavior for Asian and European-American Emerging Adults
}

\author{
Laura M. Padilla-Walker ${ }^{1}$ Larry J. Nelson ${ }^{1} \cdot$ Xinyuan Fu $^{2}$ • \\ Carolyn McNamara Barry ${ }^{3}$
}

Published online: 13 September 2017

(c) Springer Science+Business Media, LLC 2017

\begin{abstract}
The current study examined bidirectional relations between parenting and prosocial behavior for both European- and Asian-American emerging adults. Participants included 297 undergraduate students $\left(M_{\text {age }}=19.61\right.$ at Time 1, 59\% European-American) who reported on prosocial behavior toward family members, positive parenting, and negative/controlling parenting at two time points, 1 year apart. Cross-lagged models supported bidirectional relations between parenting and prosocial behavior with particular emphasis on the role of the emerging adults' prosocial behavior on subsequent parenting. Also, the bidirectional relations between parenting and emerging adults' prosocial behavior were different for mothers and fathers. Results varied slightly as a function of ethnicity. Discussion focuses on the implications for understanding the multifaceted nature of prosocial development in emerging adulthood.
\end{abstract}

Keywords Parenting · Prosocial behavior - Ethnicity · Bidirectionality

\section{Introduction}

Bioecological models of human development suggest a dynamic process in which children both influence and are

Laura M. Padilla-Walker

laura_walker@byu.edu

12071 JFSB, School of Family Life, Brigham Young University, Provo, UT 84602, USA

2 Department of Psychology, School of Sociology and Psychology, Central University of Finance and Economics, Beijing, China

3 Loyola University, Baltimore, MD, USA influenced by their environment (Bronfenbrenner and Morris 2006). It is now well understood that children are not only influenced by their parents but also directly influence their parents' behaviors (e.g., Coley et al. 2009; Eisenberg et al. 1999; Rubin et al. 1999). Most of that work has been done with younger children and adolescents but, to date, there are few if any studies that examine the bidirectional links between parenting and emerging-adult behaviors, especially prosocial behaviors, during the third decade of life. Furthermore, most of the parenting literature conducted with emerging adults has examined mothers and not fathers, and has failed to account for potential ethnic differences. In order to address these limitations, the purpose of this study was to examine longitudinally the bidirectional associations between both mothers' and fathers' parenting and prosocial behavior toward family in emerging adulthood. Furthermore, potential differences as a function of ethnicity were explored.

\section{Parenting in Emerging Adulthood}

There is a growing body of work showing that parents still play an important role in the lives of emerging adults. For example, positive aspects of parenting such as warmth, support, involvement/knowledge, autonomy granting, and communication have been linked to emerging adults' positive outcomes (e.g., kindness, self-worth, and identity development) and lower levels of negative outcomes such as risk behaviors (e.g., binge drinking, drugs, risky sexual behaviors), depression, and anxiety (e.g., Nelson et al. 2011; Padilla-Walker et al. 2008).Conversely, more negative parenting that involves high levels of control (e.g., helicopter parenting, psychological control, and behavioral control) has been linked to a myriad of problematic outcomes for 
emerging adults. For example, helicopter parenting has been found to be associated with more pessimistic views of marriage (Willoughby et al. 2015), higher levels of depression, and higher rates of prescribed medication for depression and anxiety (LeMoyne and Buchanan 2011). Behavioral and psychological control have been linked to more risk behaviors, depression, lower self-worth, and problems in identity development (e.g., Luyckx et al. 2007; Nelson et al. 2011; Urry et al. 2011). Taken together, positive parenting appears to be linked to indices of adjustment in emerging adulthood, while negative, controlling parenting is linked to indices of maladjustment. Despite the growing number of outcomes that have been studied during the third decade of life, there is a dearth of work examining prosocial behaviors (voluntary behavior meant to benefit another; Barry et al. 2008; Eisenberg et al. 2006). This is a rather significant gap in the literature given that prosocial behaviors have been identified as a distinguishing feature of emerging adults who are flourishing rather than floundering during the third decade of life (Nelson and Padilla-Walker 2013).

\section{Parenting and Prosocial Behavior}

As noted, the study of prosocial behavior in emerging adulthood has received relatively little attention despite the important role it might play as an indicator of positive development. Indeed, despite the sometimes negative perceptions of this age group, emerging adults frequently display a diversity of prosocial behaviors (Randall and Wenner 2014) and often have unique opportunities to help and serve others that were not available at previous ages and that will be more difficult at later ages (e.g., Peace Corps, Teach For America; Padilla-Walker 2015). During the third decade of life, prosocial behavior has been linked positively to prosocial values (Barry et al. 2008), sympathy (Eisenberg et al. 2002), self-esteem (Randall and Wenner 2014), and friendship quality, and negatively to substance use and deviance. Taken together, prosocial behavior is frequent during this developmental time period and is an important indicator of positive adjustment.

The majority of work on parenting and prosocial behavior has focused almost exclusively on children and adolescents, and suggests that authoritative parenting (Dekovic and Janssens 1992) and parental induction (Krevans and Gibbs 1996) are associated positively with children's display of prosocial behavior, whereas more authoritarian parenting (Hastings et al. 2000) or parenting that reflects a parent's emphasis on child compliance and obedience (Eisenberg et al. 1992) tends to be linked to lower levels of children's prosocial behavior. Although parenting has been associated with prosocial behavior toward all targets, it is particularly important in fostering prosocial behavior toward family members (Padilla-Walker and Christensen 2011). Prosocial behavior toward family members is fostered by parenting, and in turn has been found to strengthen parent-child connection and protect against future problems behaviors (Padilla-Walker et al. 2015). Thus, given the importance of parenting on prosocial behavior toward family in particular, parenting and prosocial behavior toward family will be the focus of the current study.

The work on parenting and prosocial behavior during the third decade of life is much less extensive but certainly growing. This work can be divided into two types. First, there is a small but important body of literature focusing on the association between the parent-child relationship (e.g., relationship quality, attachment) and prosocial behaviors (e.g., Laible et al. 2004). For example, the quality of the mother-child relationship has been found to be related to emerging adults' internalization of values, which, in turn, is associated with prosocial behavior (Barry et al. 2008). In the second emerging line of work, specific aspects of parenting practices have been linked to emerging adults' prosocial behaviors. For example, it has been found that retrospective accounts of emerging adults' perceptions of mothers' (but not fathers') authoritative parenting predicted more prosocial engagement and a stronger sense of self as being empathic (Soucie et al. 2012). Also, Roth (2008) found that young people's perception of parental conditional regard (i.e., belief that receiving their parents' affection was dependent on their behaving prosocially towards others) was associated with young people's participation in self-oriented helping behaviors while perceived parental autonomy support was linked to young people's participation in other-oriented behaviors. Taken together, this growing body of work suggests that parents do indeed play a role in the extent to which their emerging-adult children engage in prosocial behaviors but there is still much to be learned about the exact nature of that parental role, especially as it is specifically associated with prosocial behavior toward family members.

\section{Current Study}

As noted, this extant work has provided a strong foundation for our understanding the links between parenting and prosocial behaviors during the third decade of life, but there are numerous limitations to past work that need addressing. First, there have been a number of retrospective and longitudinal studies (e.g., Masten et al. 2004; Smits et al. 2008) but few if any longitudinal studies conducted throughout the third decade of life and, none, that we are aware of examining prosocial behaviors. As a result, the majority of work fails to examine the potential bidirectional effects of both parenting and child behaviors/characteristics. Specifically, we do not know if parenting predicts an increase in emerging 
adults' display of prosocial behaviors, or if emerging adults' participation in positive, prosocial behaviors elicits positive parental behaviors.

Recent work with children has demonstrated the need to examine the bidirectional effects of parenting and prosocial behaviors. In a longitudinal study of young children, evidence for bidirectional effects were found with children's prosocial behavior predicting subsequent maternal sensitivity even when controlling for concurrent prosocial behavior and past parental sensitivity (Newton et al. 2014). This provides evidence for bidirectionality and supports the logic that both parents' and emerging adults' behaviors would be impacting each other given that this is a period of time in which both parents and emerging adults are striving to adjust the nature of their relationship (e.g., Arnett 2014; Aquilino 1997, 2006). Thus, the first purpose of this study was to examine the bidirectional associations between parenting (both positive and negative aspects of parenting) and prosocial behavior in emerging adulthood. Based on research during childhood (Newton et al. 2014), and work in adolescence that suggests more consistent paths from child behavior to parenting rather than the other way around (Moilanen et al. 2015; Padilla-Walker et al. 2012), we expected there to be bidirectional paths between parenting and prosocial behavior, but thought it particularly likely that the emerging-adult child's behavior would be linked to later parenting.

A second weakness of the small body of existing research is that much of it has simply examined the extent to which prosocial behaviors have been performed generally (i.e., without examining the target of the behavior; e.g., Barry et al. 2008; Roth 2008) or has focused on prosocial behavior directed at strangers or peers. Although important to examine prosocial behavior towards peers and strangers given the increasing amount of time spent outside of the family in emerging adulthood, more attention is being given to the importance of studying prosocial behavior in the home or family context (e.g., Eberly and Montemayor 1998; PadillaWalker and Christensen 2011; Padilla-Walker et al. 2012). We felt this was especially important given that emerging adulthood is a time in which young people are striving to develop a relationship with parents that is more peer-like (e.g., Arnett 1998, 2014). Furthermore, as young people leave adolescence, there should be an increase in autonomy and self-direction from parents (e.g., Arnett 2014; Aquilino 2006; Wartman and Savage 2008) and, therefore, any prosocial behavior towards family should be truly more voluntary in nature and play a significant role in the renegotiation of the parent-child relationship that is taking place. Finally, with many emerging adults living outside the parental home and spending increased amounts of time with peers, parents may not see as many prosocial behaviors towards non-family members (e.g., strangers, peers) as at early ages and, therefore, prosocial behavior towards others might not elicit as strong a reaction from parents as would prosocial behaviors towards family members. Thus, in examining the bidirectional effects of emerging adults' prosocial behaviors and parenting, we thought it would be prosocial behavior toward family that would be the most relevant target to examine.

The growing body of work underscores the need for more work to be done examining both mothers and fathers when examining parenting and emerging adults' prosocial behaviors. Research examining the role of parents, generally, in emerging adulthood shows that fathers hold different expectations for emerging adults (e.g., Nelson et al. 2007), parent differently than do mothers and, as a result, impact their children's outcomes in unique ways from those of mothers (e.g., Nelson et al. 2011). Taken together, we deemed it important to examine parenting of both mothers and fathers to examine how they might affect and/or be affected by their emerging-adult children's prosocial behaviors. Based on research during childhood and adolescence suggesting that positive mothering is positively associated with prosocial behavior while negative fathering is negatively associated with prosocial behavior (Dekovic and Janssens 1992; Padilla-Walker et al. 2014; Perez-Albeniz and de Paul 2004), and that children's prosocial behavior predicted subsequent maternal (but not paternal) sensitivity, we hypothesized that this same pattern would exist in emerging adulthood as well.

Finally, there has been very little work examining the role of culture or ethnicity in the link between parenting and prosocial behaviors during emerging adulthood. One notable exception is the work done by Carlo and colleagues who found that parent-child attachment was positively related to prosocial behaviors in a sample of Mexican-American college students (Carlo et al. 2011). Although there was no cross-culture comparisons conducted in their work, it does suggest that culture might impact parental attempts to foster certain desired behaviors. Building on this, we believe that children as well as their parents might interpret one another's behavior through the lens of the cultural values associated with those behaviors.

In particular, many Asian families identify with a value system that is characterized as being more collectivistic while a value system that places emphasis on the individual tends to be embraced by many families and individuals in the United States. However, both value systems may be influencing developmental processes for Asian-American families especially if the parents were born in Asia but their children were born in the United States. ${ }^{1}$ There is certainly evidence

\footnotetext{
$\overline{1}$ It should be noted from the outset that Asian-American families come from a wide variety of Asian countries capturing a plethora of unique and distinct cultures and belief systems. However, much of the extant work has focused on China and Chinese-Americans so we will draw extensively from this literature while, again, acknowledging the extreme diversity represented in Asian-American families and communities.
} 
to suggest differences in parenting between Asian-American and European-American parents. Chinese-American parents have been found to be somewhat higher on controlling/authoritarian parenting than are European-American parents (Chao 2000). This controlling/authoritarian parenting encompasses a range of aspects, such as controlling children's use of time outside of school, purchasing extra textbooks, assigning extra homework from these textbooks, enrolling them in music or language lessons, and arranging for them to help with homework (e.g., Ho and Willms 1996). Deeply rooted in Confucianism, the tradition of Chinese parenting involves training children early through guidance and continuous monitoring of their behaviors, and emphasizes obedience and a set standard of conduct (Chao 1994, 2000). As previous research shows, Chinese mothers who adhered most strongly to the Chinese cultural values (based on Confucian beliefs) had high scores of authoritarian parenting styles (Xu et al. 2005).

It should be pointed out, though, that both children and parents may experience frustration when comparing their own experience with that of European-Americans (Qin 2006, 2008). Asian-American emerging adults may compare and feel that their parents are unfair, while parents may continue to interpret their more Westernized children through their collectivistic values. Thus, there may be even more complexity in the bidirectionality of the parent-child relationship in emerging adulthood for Asian-American families. In particular, prosocial behavior in general and prosocial behavior towards family specifically may be especially relevant and complex for Asian-American families. A prosocial orientation is highly valued and encouraged in many Asian-American families since it is essential for the functioning of the collective (Ho 1986). Indeed, in some of the limited work on the topic, prosocial behavior appears to have great significance for Chinese emerging adults as it has been positively related to socioemotional variables, including interpersonal interaction, perceived self-worth, and life meaningfulness (Jin et al. 2013), as well as activity involvement (social, educational, and entertainment activities) and educational attainment (Chen et al. 2002).

In sum, prosocial behavior toward family may mean something different for Asian-American emerging adults and their parents than for European-American emerging adults in that it is driven by different cultural values. Confucianism teaches the concept of Filial Piety, with the emphasis of respecting the elders and honoring the family by performing filial behavior (Yao 2000). Therefore, Asian-American parents may expect their children to engage in prosocial behaviors towards family, and emerging adults might feel more obligated to engage in prosocial behaviors towards family than do their European-American peers. For this reason, the current study examined bidirectional relations between mothering and fathering and prosocial behavior toward family for both European- and Asian-American emerging adults. Because of the relative dearth of research as a function of ethnicity during this age period, our analyses in this regard were exploratory.

\section{Methods}

\section{Participants}

Participants for this study were drawn from a study of emerging adults entitled Project READY (Researching Emerging Adults' Developmental Years). This project is a collaborative, multi-site study that was conducted by a consortium of developmental and family scholars. Time 1 data were collected during 2009-2010 and approximately one year later for Time 2 data. The sample for the current study $\left(M_{\text {age }}=19.61, S D=1.92\right.$, range $18-28$ at Time 1$)$ consisted of 297 undergraduate students (50\% female). Participants were recruited from two public 4-year universities in the Western and Midwestern United States. Response rate varied by site, with an initial response rate of approximately $60 \%$ and a longitudinal response rate of 55\%. Missing data were handled using the Full Information Maximum Likelihood feature of AMOS (Arbuckle 2010).

In terms of year in school, $42 \%$ of emerging adults were in their first year, 27\% second year, 17\% third year, and $11 \%$ fourth year. Ninety-one percent of emerging adults reported living outside of their parents' home in an apartment, house, or dormitory. Thirty-four percent reported that their parents' combined income was $\$ 50,000$ per year or less, and $19 \%$ reported combined parental income over $\$ 100,000$. Fiftynine percent of the sample described themselves as European-American, while $41 \%$ described themselves as AsianAmerican $(n=121)$. Seventy percent of Asian-American emerging adults reported being born in the United States, but only $6 \%$ reported having parents who were born in the United States.

\section{Procedure}

Participants completed the Project READY questionnaire via the Internet. The use of an online data collection protocol facilitated unified data collection across multiple university sites. At Time 1, participants were recruited through faculty's announcement of the study in undergraduate courses. Undergraduate courses were primarily Introduction to Psychology courses or large general education courses of the like in an attempt to access a broad range of students. Professors at the various universities were provided with a handout to give to their students that had a brief explanation of the study (i.e., a study about development among college students) and directions for accessing the online 
survey. Interested students then accessed the study website with a class-specific recruitment code. Informed consent was obtained online, and each participant was given a survey that took approximately $45 \mathrm{~min}$ to complete. Participants were given a \$20 Amazon gift code for their participation. At Time 2, participants were contacted via e-mail and given a link to the same survey questions administered at Time 1 . Participants were given a $\$ 50$ Amazon gift code for their participation at Time 2.

\section{Measures}

\section{Prosocial Behavior Toward Family Members}

Prosocial behavior toward family members was examined at Time 1 and 2 using five items $(\alpha=0.91,0.93)$ from a modified version of the kindness/generosity subscale of the values in action inventory of strengths (Peterson and Seligman 2004). Items were modified to target prosocial behavior toward family members (as opposed to unspecified targets in the original measure) and were adapted to be appropriate for this age group. This measure has been validated in previous studies with adolescents (Padilla-Walker et al. 2012). Participants responded to items on a 5-point Likert scale ranging from 1 (not like me at all) to 5 (very much like me). Sample questions include, "I really enjoy doing small favors for my family," and "I help my family, even if it is not easy for me."

\section{Positive Parenting}

Positive parenting was assessed at Time 1 and 2 using the warmth, involvement, and autonomy support subscales of the perception of parents scale (POPS): college student version (Grolnick et al. 1991; Robbins 1994). Emerging adults answered questions on a 7-point scale ranging from 1 (not at all true) to 7 (very true). Sample questions include, "My parent finds time to talk with me" for warmth (6 items, childreport mother, $\alpha=0.86,0.88$; child-report father, $\alpha=0.89$, 0.88 ), "My parent spends a lot of time with me" for involvement ( 6 items, child-report mother, $\alpha=0.80,0.86$; childreport father, $\alpha=0.85,0.88$ ), and "My parent helps me to choose my own direction" for autonomy support (8 items, child-report mother, $\alpha=0.85,0.87$; child-report father, $\alpha=$ $0.87,0.91)$.

\section{Negative/Controlling Parenting}

Behavioral control was assessed at Time 1 and 2 using five items assessing parents' tendency to control their child's friends, money, or activities (Kerr and Stattin 2000). Emerging adults answered questions on a 5-point scale ranging from 1 (not at all like him/her) to 5 (a lot like him/her). Sample items include, "My parent tries to set rules about what I do with my free time," and "My parent tries to tell me what I can and can't do on nights and weekends." Higher scores indicated higher levels of child-reported mother $(\alpha=0.89$, $0.84)$ and father $(\alpha=0.87,0.82)$ behavioral control.

Psychological control was assessed at Time 1 and 2 using four items assessing psychologically controlling parenting (Barber 1996). Emerging adults answered questions on a 3-point scale ranging from 1 (not at all like him/her) to 3 (a lot like him/her). Sample items include, "If I have hurt his/ her feelings, my parents stops talking to me until I please him/her again," and "My parent will avoid looking at me when I have disappointed her/him." Higher scores indicated higher levels of child-reported mother $(\alpha=0.83,0.86)$ and father $(\alpha=0.81,0.88)$ psychological control.

\section{Results}

\section{Descriptive Statistics and Correlations}

A number of $t$-tests were used to determine whether variables differed as a function of ethnicity, and the majority of these analyses were statistically significant (Table 1). In general, it should be noted that Asian-American emerging adults reported lower levels of positive maternal and paternal parenting and higher levels of negative parenting than did European-American emerging adults. However, there were no differences in prosocial behavior as a function of ethnicity at either time point. Tables 2 and 3 contain bivariate correlations between parenting and prosocial behavior, separately for Asian and European-Americans. It should be noted that correlations were similar across ethnicity save for no significant relations between positive parenting at Time 1 and prosocial behavior at Time 2 for European-Americans (relations were significant for Asian-Americans).

\section{Measurement Invariance}

Because the sample size was too small to examine the entire model using latent variables, we examined measurement invariance for each latent construct separately. This was done to examine invariance by time point and then by ethnicity. Only weak (factor loadings) and strong (intercepts) invariances are needed to compare means across time or groups (Little 2013), so these two were tested. First, latent variables were created (separate for each construct) in a fully unconstrained model. To examine weak invariance, factor loadings were constrained to be equal (across time and then ethnicity) and invariance was achieved if the CFI did not decrease more than 0.01 (Little 2013). In terms of invariance over time, CFI changes as a result of constraining factor loadings ranged from 0.001 to 0.006 , and in terms of variance across ethnicity CFI changes ranged from 0.00 
Table 1 Means and standard deviations as a function of ethnicity

\begin{tabular}{|c|c|c|c|c|}
\hline & $\begin{array}{l}\text { Asian- } \\
\text { American } \\
M(S D)\end{array}$ & $\begin{array}{l}\text { European- } \\
\text { American } M \\
(S D)\end{array}$ & $t$-test & $d$ \\
\hline M involvement (1) & $5.23(1.12)$ & $5.69(1.06)$ & $-3.61 * * *$ & 0.42 \\
\hline $\mathrm{P}$ involvement (1) & $4.90(1.06)$ & $5.25(1.31)$ & $-2.47 *$ & 0.29 \\
\hline M autonomy (1) & $4.96(1.03)$ & $5.59(1.11)$ & $-4.99 * * *$ & 0.59 \\
\hline P autonomy (1) & $4.64(1.10)$ & $5.37(1.15)$ & $-5.51 * * *$ & 0.65 \\
\hline M warmth (1) & $5.60(1.09)$ & $6.02(1.14)$ & $-3.21 * *$ & 0.38 \\
\hline P warmth (1) & $5.39(1.19)$ & $5.85(1.20)$ & $-3.23 * * *$ & 0.38 \\
\hline M Beh control (1) & $2.49(0.99)$ & $1.97(0.94)$ & $4.60 * * *$ & 0.54 \\
\hline P Beh control (1) & $2.35(1.03)$ & $1.86(0.81)$ & $4.56 * * *$ & 0.53 \\
\hline M Psy control (1) & $1.67(0.59)$ & $1.40(0.52)$ & $4.16^{* * *}$ & 0.49 \\
\hline P Psy control (1) & $1.59(0.62)$ & $1.39(0.50)$ & $3.03 * *$ & 0.36 \\
\hline PB (1) & $4.10(0.85)$ & $4.16(0.70)$ & -0.71 & 0.08 \\
\hline M involvement (2) & $5.21(1.23)$ & $5.73(1.05)$ & $-2.69 * *$ & 0.45 \\
\hline $\mathrm{P}$ involvement (2) & $4.68(1.43)$ & $5.31(1.30)$ & $-2.91 * *$ & 0.46 \\
\hline M autonomy (2) & $5.01(1.18)$ & $5.65(1.02)$ & $-3.75^{* * *}$ & 0.58 \\
\hline P autonomy (2) & $4.40(1.30)$ & $5.34(1.12)$ & $-5.02 * * *$ & 0.77 \\
\hline M warmth (2) & $5.61(1.13)$ & $6.12(1.04)$ & $-3.04 * *$ & 0.47 \\
\hline P warmth (2) & $5.17(1.47)$ & $5.94(1.01)$ & $-4.06 * * *$ & 0.61 \\
\hline $\begin{array}{l}\text { M Beh CONTROL } \\
\text { (2) }\end{array}$ & $2.17(0.91)$ & $1.68(0.69)$ & $4.03 * * *$ & 0.61 \\
\hline P Beh control (2) & $1.97(0.78)$ & $1.64(0.68)$ & $2.88 * *$ & 0.45 \\
\hline M Psy control (2) & $1.46(0.50)$ & $1.42(0.55)$ & 0.57 & 0.08 \\
\hline P Psy control (2) & $1.52(0.61)$ & $1.35(0.49)$ & $2.07 *$ & 0.31 \\
\hline PB (2) & $4.08(0.84)$ & $4.25(0.66)$ & -1.48 & 0.23 \\
\hline
\end{tabular}

$M$ maternal, $P$ paternal, $P B$ prosocial behavior, 1 Time 1, 2 Time 2

${ }^{*} p<.05, * * p<.01, * * * p<.001$

to 0.003 . Because none of these changes were $>0.01$, we determined that weak invariance was established. Next, to examine strong invariance, intercepts were constrained to be equal (across time and ethnicity) and were compared to the weak invariant model. In terms of invariance over time, CFI changes as a result of constraining intercepts ranged from 0.00 to 0.003 , and in terms of variance across ethnicity CFI changes ranged from 0.01 to 0.02 . The changes that were $>0.01$ were for parental warmth and behavioral control. Thus, individual items were examined and partial strong invariance was established by freeing constraints on one maternal warmth item ("My parent clearly conveys his/ her love for me," which was higher for European-Americans) and one behavioral control item ("My parent tries to limit or control who my friends are") which was higher for Asian-Americans.

\section{Cross-Lagged Models for Prosocial Behavior Toward Family}

Cross-lagged models were conducted using AMOS software (Arbuckle 2010), and were conducted separately for perceptions of maternal and paternal parenting because of collinearity between emerging adults' reports of mothers and fathers, which resulted in suppression effects when parenting variables were all included in the same model. Model fit was considered acceptable with CFI $>0.90$, TLI $>0.90$, and RMSEA $<0.08$ (Little 2013). All models used the child's gender $(0=$ male, $1=$ female $)$ and generational status $(0=1$ st generation, $1=2$ nd generation $)$ as controls. Multiple group comparisons were conducted on all models as a function of ethnicity, comparing a model where all paths were constrained to be equal across Asianand European-Americans to a model where all paths were freely estimated. If decrease in model fit was determined by $\Delta \chi^{2}$ analyses, paths were examined one at a time to determine which paths were contributing to the decrease in model fit. Those paths were then left free to vary and all others were constrained to be equal.

\section{Maternal Parenting}

The first model examined cross-lagged paths between mothers' positive (involvement, autonomy granting, warmth) and negative (behavioral and psychological control) parenting and prosocial behavior toward family at Time 1 and Time 2 (see Fig. 1). Variables at Time 1 were correlated with one another, as were residual errors at Time 2. A multiple group comparison $\Delta \chi^{2}$ test suggested that constraining the pathways to be equal across ethnic groups did not result in a decrease in model fit $\left(\Delta \chi^{2}(16)=19.52\right.$, ns. $)$, so a single group model was used as the final model and had good model fit $\left(\chi^{2}(24)=51.47, p<.01, \mathrm{CFI}=0.99, \mathrm{TLI}=0.97\right.$, RMSEA $=0.06$ ). Results suggested that prosocial behavior toward family at Time 1 was positively associated with maternal autonomy granting $(\beta=.0 .08, p<.05)$ and warmth $(\beta=0.13, p<.001)$ at Time 2 (see Fig. 1$)$; and negatively associated with behavior control $(\beta=-0.12, p<.01)$ at Time 2 . Prosocial behavior at Time 1 was not significantly associated with parental involvement $(\beta=.03, \mathrm{~ns}$.) or psychological control $(\beta=-0.03$, ns.) at Time 2 ; nor was parental involvement ( $\beta=-0.06, \mathrm{~ns}$.), autonomy granting $(\beta=-.05$, ns.), warmth ( $\beta=.05$, ns.), behavioral control ( $\beta=0.05$, ns.), or psychological control $(\beta=.00$, ns. $)$ at Time 1 associated with prosocial behavior at Time 2 . In terms of controls, generation status was positively correlated with parental warmth $(\beta=.08, p<.01)$. All stability paths were also significant from Time 1 to Time $2(p<.001)$. All exogenous variables were correlated at Time 1 , with correlations between positive parenting ranging from .75 to .80 , a correlation between negative of .50 , correlations between positive and negative parenting ranging from -.53 to -.15 , and correlations between parenting and prosocial behavior ranging from -.14 to .50 . 


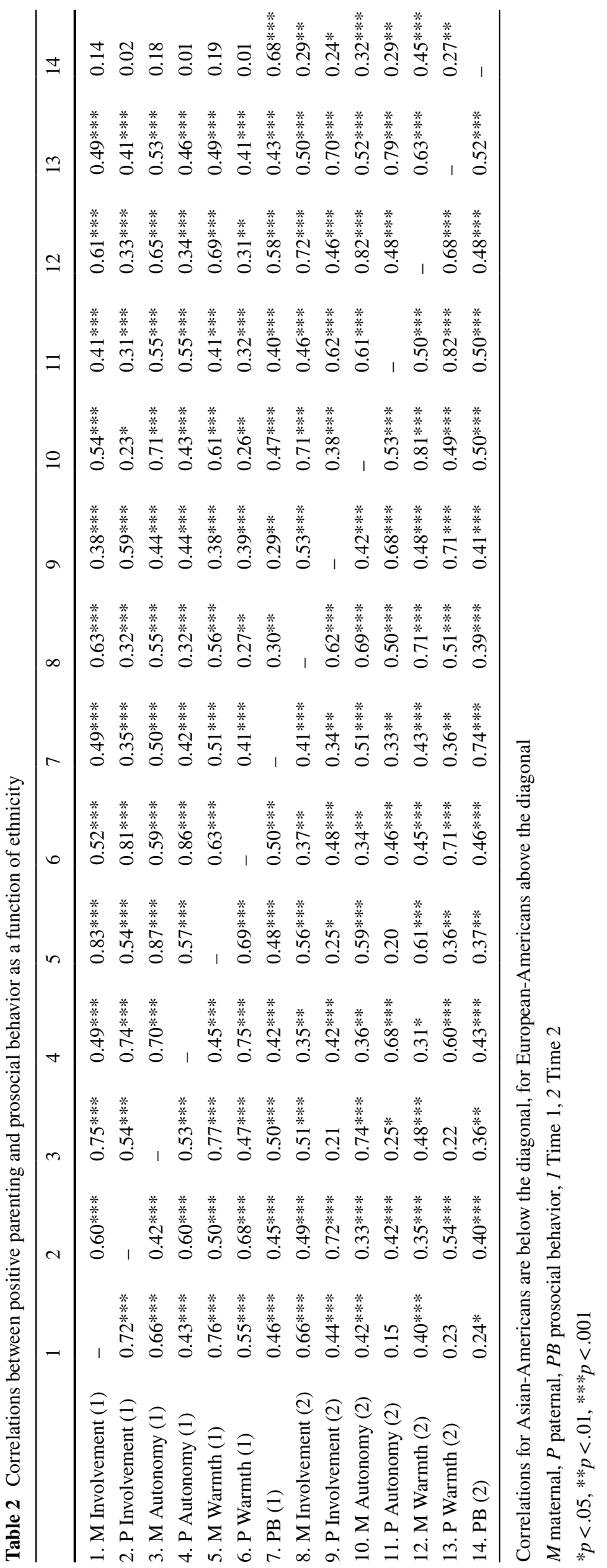


Table 3 Correlations between negative parenting and prosocial behavior as a function of ethnicity

\begin{tabular}{|c|c|c|c|c|c|c|c|c|c|c|}
\hline & 1 & 2 & 3 & 4 & 5 & 6 & 7 & 8 & 9 & 10 \\
\hline 1. M Beh control (1) & - & $0.76^{* * *}$ & $0.39 * * *$ & $0.24 * * *$ & $-0.22 * * *$ & $0.52 * * *$ & $0.40 * * *$ & $0.25^{*}$ & $0.25^{*}$ & 0.07 \\
\hline 2. P Beh control (1) & $0.66^{* * *}$ & - & $0.23 * *$ & $0.24 * *$ & -0.07 & $0.49 * * *$ & $0.54 * * *$ & 0.06 & $0.23 *$ & 0.04 \\
\hline 3. M Psy control (1) & $0.46^{* * *}$ & 0.17 & - & $0.33 * * *$ & $-0.26^{* * * *}$ & 0.18 & $0.21 *$ & $0.71 * * *$ & $0.25^{*}$ & -0.15 \\
\hline 4. P Psy control (1) & 0.14 & $0.43 * * *$ & $0.32 * * *$ & - & -0.12 & $0.27 * *$ & $0.37 * * *$ & 0.09 & $0.46 * * *$ & 0.14 \\
\hline 5. PB (1) & -0.08 & -0.02 & -0.16 & -0.16 & - & $-0.34 * * *$ & $-0.32 * * *$ & $-0.32 * * *$ & -0.11 & $0.68 * * *$ \\
\hline 6. M Beh control (2) & $0.46^{* * *}$ & $0.30 *$ & $0.42 * * *$ & 0.11 & $-0.25^{*}$ & - & $0.81 * * *$ & $0.26 * *$ & $0.41 * * *$ & -0.11 \\
\hline 7. P Beh control (2) & $0.21 *$ & $0.61 * * *$ & 0.12 & $0.37 * *$ & -0.03 & $0.47 * * *$ & - & $0.24 *$ & $0.48 * * *$ & -0.05 \\
\hline 8. M Psy control (2) & $0.40 * * *$ & 0.06 & $0.67 * * *$ & 0.19 & -0.12 & $0.45 * * *$ & -0.03 & - & $0.47 * * *$ & $-0.23^{*}$ \\
\hline 9. P Psy control (2) & 0.18 & $0.42 * * *$ & 0.22 & $0.60 * * *$ & -0.22 & 0.19 & $0.27 *$ & $0.25^{*}$ & - & 0.04 \\
\hline 10. PB (2) & -0.20 & -0.07 & -0.15 & -0.02 & $0.74 * * *$ & -0.22 & -0.07 & -0.10 & -0.16 & - \\
\hline
\end{tabular}

Correlations for Asian-Americans are below the diagonal, for European-Americans above the diagonal

$M$ maternal, $P$ paternal, $P B$ prosocial behavior, 1 Time 1, 2 Time 2

${ }^{*} p<.05, * * p<.01, * * * p<.001$

Fig. 1 Cross-lagged effects of maternal parenting and prosocial behavior toward family. Note all paths represent significant standardized beta weights. Control variables, correlations between variables at Time 1 , and residual covariances at Time 2 were omitted from figure for parsimony. $* p<.05$, $* * p<.01, * * * p<.001$

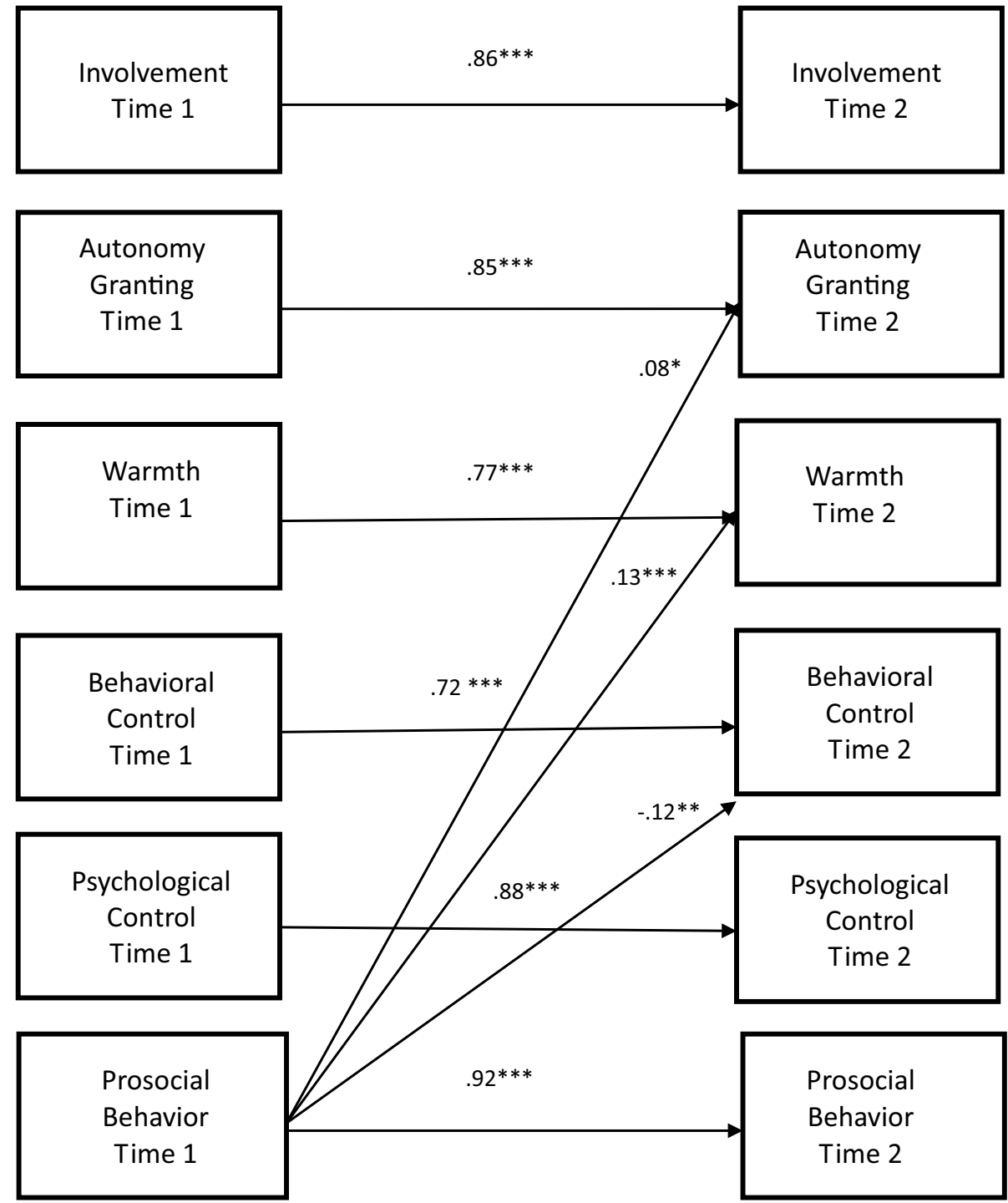


Fig. 2 Cross-lagged effects of paternal parenting and prosocial behavior toward family. Note all paths represent significant standardized beta weights, values before the slash are for Asian-Americans and after the slash are for European-Americans. Dashed lines represent paths that were unconstrained between groups. Control variables, correlations between variables at Time 1 , and residual covariances at Time 2 were omitted from figure for parsimony. $* p<.05, * * p<.01$, $* * * p<.001$

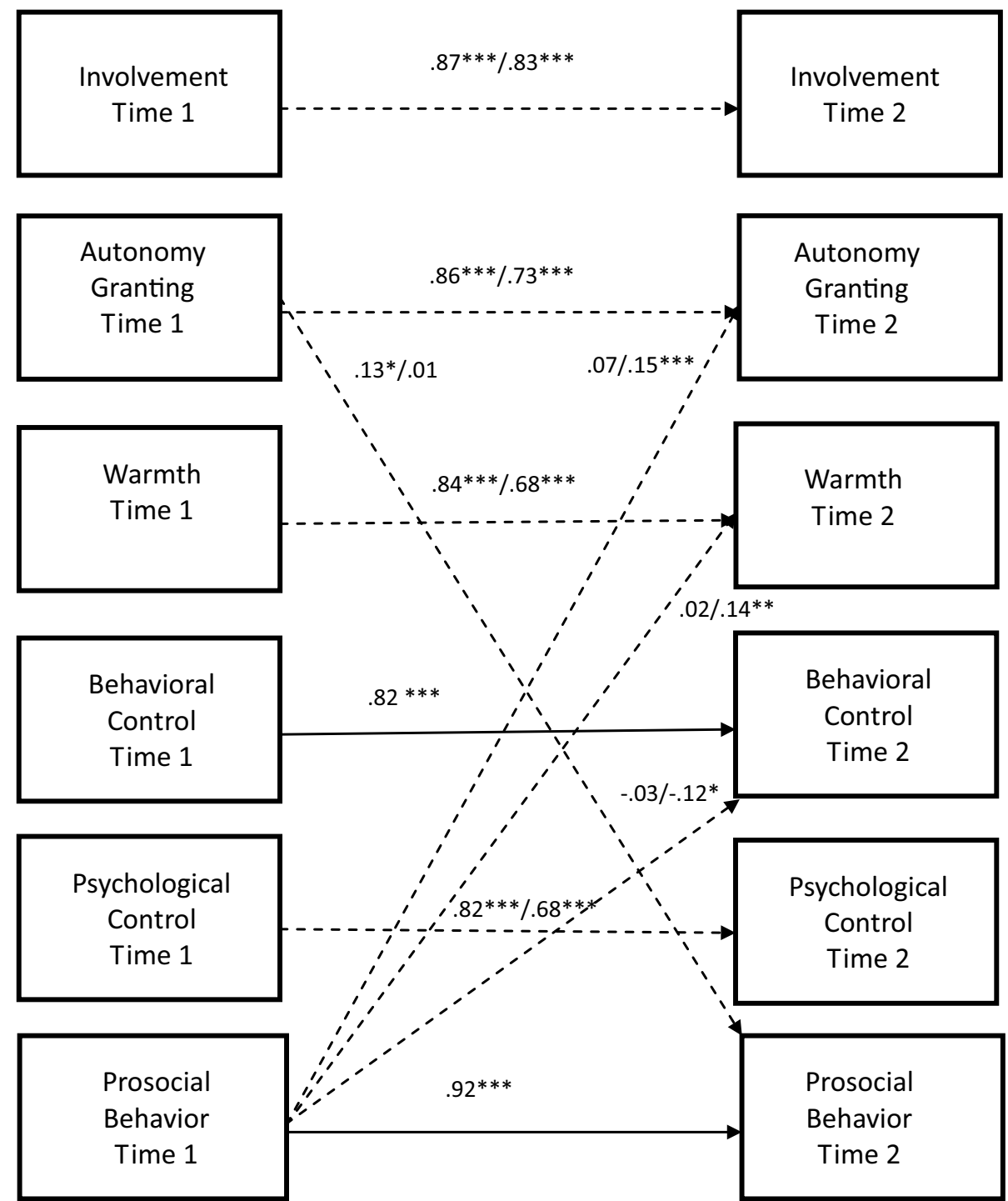

\section{Paternal Parenting}

The second model examined cross-lagged paths between fathers' positive (involvement, autonomy granting, warmth) and negative (behavioral and psychological control) parenting and prosocial behavior toward family at Time 1 and Time 2 (see Fig. 2). A multiple group comparison $\Delta \chi^{2}$ test suggested that constraining all pathways to be equal across ethnic groups resulted in a decrease in model fit $\left(\chi^{2}(16)=52.56\right.$, $p<.001$ ), so follow-up analyses were conducted freeing one path at a time and constraining only those paths that did not result in a decrease in model fit per $\Delta \chi^{2}$. The paths that were left free to vary included stability paths for parental involvement, autonomy, warmth, and psychological control; as well as structural paths from autonomy at Time 1 to prosocial behavior at Time 2 and prosocial behavior at Time 1 to autonomy granting, warmth, and behavioral control at Time 2. All other paths were constrained to be equal. The final multiple group model had good model fit $\left(\chi^{2}(55)=94.52\right.$, $p<.01, \mathrm{CFI}=.99, \mathrm{TLI}=.96$, RMSEA $=0.05)$. Results suggested that for Asian-Americans, paternal autonomy granting at Time 1 was positively associated with prosocial behavior toward family at Time $2(\beta=0.13, p<.05)$, while for European-Americans prosocial behavior toward family at Time 1 was positively associated with both paternal autonomy granting $(\beta=0.15, p<.001)$ and warmth $(\beta=0.14$, $p<.01)$ at Time 2 , and negatively associated with behavior control $(\beta=-0.12, p<.05)$ at Time 2 . Prosocial behavior at Time 1 was not significantly associated with parental involvement for Asian- or European-Americans $(\beta=.04 / .03$, ns.) or psychological control $(\beta=-.08 /-.07, \mathrm{~ns}$. $)$ at Time 2 ; nor was parental involvement $(\beta=-.03 /-.04$, ns. $)$, warmth ( $\beta=-.04 /-.04$, ns. $)$, behavioral control $(\beta=0.00 / .00, \mathrm{~ns}$.$) ,$ or psychological control $(\beta=.03 / .03$, ns.) at Time 1 associated with prosocial behavior at Time 2 . In terms of controls, generation status was positively correlated with parental 
warmth $(\beta=.09, p<.01)$. It should also be noted that all stability paths were significant $(p<.001)$ but four differed significantly as a function of ethnicity (see Fig. 2). All exogenous variables were correlated at Time 1 , with correlations between positive parenting ranging from .73 to .81 , a correlation between negative of .51 , correlations between positive and negative parenting ranging from -.27 to .23 , and correlations between parenting and prosocial behavior ranging from -0.22 to 0.46 .

\section{Discussion}

With the transition to adulthood taking longer and longer for many young people in Western cultures such as the United States (see Arnett 2000), it appears that parents are continuing to play a significant role in the lives of their children during the third decade of life (e.g., Nelson and Padilla-Walker 2013). However, there has been very little longitudinal work examining (a) associations between parenting and prosocial behaviors, (b) the bidirectional effects of parenting (including examining both mothers and fathers) and young people's prosocial behavior (particularly towards family), and (c) the potential role of ethnicity in these linkages. Thus, the purpose of this study was to examine longitudinally the bidirectional associations between both mothers' and fathers' parenting and prosocial behaviors toward family in Asianand European-American emerging adults.

\section{The Role of Emerging-adult Children in Influencing Parents}

The first significant contribution that the current findings make to our understanding of parenting emerging adults is to underscore the bidirectional nature of the parent-child relationship during the third decade of life, especially in terms of the role of the child's impact on parenting. While research on parenting during the formative years suggests bidirectional relations between parenting and children's behavior (e.g., Coley et al. 2009; Eisenberg et al. 1999; Fish and Crockenberg 1986; Newton et al. 2014; PadillaWalker et al. 2012; Rubin et al. 1999), the present study suggests the most consistent evidence favoring the role of emerging-adult children's behaviors on subsequent parenting. Specifically, results suggest that as emerging adults engage in more prosocial behavior towards family members, parents in turn appear to be warmer toward and grant more autonomy to their children. In discussing these findings, it should be noted from the outset that there was a high level of stability in both parenting and prosocial behavior across time and the effects of prosocial behavior on parenting were low in magnitude suggesting, among other things, that the extent to which young people engage in prosocial behavior towards family (and its pattern of interaction with parenting), might be well established by emerging adulthood. For example, similar findings have been found in adolescence (Padilla-Walker et al. 2012) suggesting that a trend might start in adolescence (and likely earlier) and continue into emerging adulthood in which parents respond to their children's positive behavior in kind, with more positive behavior themselves. So while the modest magnitude of effects gives us reason to not overstate the meaning of the findings, the results nevertheless highlight the importance of prosocial behavior for this age group as not only being an indicator of general social competence (prosocial behavior toward peers) or of community service (prosocial behavior toward strangers), but also as a potentially important tool in strengthening parent-child relationships during a time of transition.

Indeed, it is important to underscore that parental control appears to decrease and autonomy granting and warmth increase as children display more positive behavior. This is important because emerging adulthood, in particular, is a time during which reorganization of the parent-child relationship needs to take place because of the necessity for and requisite development of increased autonomy and self-direction in the lives of young people (e.g., Arnett 2000; Aquilino 2006; Wartman and Savage 2008). Becoming a self-reliant individual is seen by both young people and their parents as essential in the process of becoming an adult (e.g., Nelson et al. 2007). However, findings from the present study might suggest that parents may be waiting for evidence in their children's behavior suggesting that they warrant greater levels of autonomy pointing to the possibility that at least some of what occurs within the parent-child relationship during this time rests upon the actions of the child.

\section{The Role of Ethnicity}

The other important contribution that these results make pertains to the role of cultural beliefs and values in parenting and emerging adults' behavior. With the exception of work by Carlo and colleagues (2012), there is very little work examining parenting differences during emerging adulthood in ethnic sub-groups within the United States. Thus, our exploration of possible differences between Asian-American and European-American was an attempt to address this gap in the literature. Surprisingly, European-American and Asian-American mothers did not differ in regard to the bidirectional links between parenting and emerging adults' prosocial behavior. There were, however, significant differences in the associations between parenting and prosocial behavior for Asian-American fathers and their EuropeanAmerican peers. Specifically, Asian-American fathers' autonomy supportive parenting at Time 1 led to increases in their children's prosocial behavior at Time 2, while there was no evidence of the influence of fathering on subsequent 
prosocial behavior for European-Americans. Furthermore, European-American emerging adults' prosocial behaviors at Time 1 predicted paternal positive and controlling parenting at Time 2, but this was not the case for Asian-Americans. These findings are somewhat unexpected and will be discussed in turn.

First, the results add to a small but growing body of work suggesting that fathers might play a particularly important role in the development of prosocial behaviors in Asian families. Studies have found paternal warmth to be positively associated with Chinese college students' prosocial behavior (e.g., Wu 2009). Also, father involvement had a positive impact on children's prosocial behavior, and father-child attachment significantly mediated the relation between father involvement and children's prosocial behavior ( $\mathrm{Li}$ et al. 2012). Employing a longitudinal design and examining bidirectional effects, the results of the present study are important because they highlight the fact that paternal autonomy granting in particular may have a small impact on the development of prosocial behavior in Asian-American emerging adults. The reason for this may stem from the fact that parental behavior that is autonomy granting as opposed to controlling/authoritarian might be particularly salient to young people in Asian cultures. Typically, Chinese parents are less likely to use low-power inductive parenting and more likely to endorse restrictive and high-power approaches (e.g., Chao 2000). Indeed, Asian-American children tend to see their parents as being much more controlling than the parents of their European-American peers (Qin et al. 2008). Hence, if parents, especially fathers, allow for greater autonomy, their emerging-adult children might reciprocate with positive behaviors of their own directed towards their family. This may very well be a case in which positive behaviors elicit positive behaviors from others especially in a setting in which the behaviors of fathers stand out in rather stark contrast to what is often seen from fathers within the Asian cultural context.

Second, it is interesting that the results revealed that emerging adults' prosocial behavior at Time 1 predicted parental behaviors at Time 2 for European-Americans but not Asian-Americans. There are a number of cultural factors that might need to be considered in order to account for these findings. For example, as noted previously, on average, Asian parents tend to be more controlling in their parenting and, therefore, they may be affected less by their children's behavior. Although this is possible, it may be more likely that Asian-American parents do not see prosocial behavior towards family as exceptional in any way as it is a cultural expectation that children of any age act that way towards family. Due to obligations associated with Filial Piety, children may be expected to treat their families well out of a sense of duty (Hwang 1999). Hence, when prosocial behavior towards family occurs in European-American families, parents might see it as a sign of increasing maturity in their emerging-adult children and respond with less control and greater warmth and autonomy but when it occurs in an Asian-American home it is expected and therefore provides no impetus for change in parental behavior. Future work should certainly explore these and other possibilities in order to account for the cultural differences seen in our results in the impact of emerging adults' prosocial behavior on European-American parents but not Asian-American parents.

\section{Limitations and Conclusions}

It should be noted that this study was not without limitations. Most notably, the sample consisted of college students, and therefore, may not be generalizable to a non-student population. There is still relatively little that is known about individual differences of those who do not attend college after high school. Therefore, it is possible that the bidirectional links between parenting and emerging adults' prosocial behaviors may work differently for those who do not attend college and their parents.

Another limitation to this study is the way in which we broadly defined and categorized Asian-Americans. As noted previously, Asian-American families come from a wide variety of Asian countries capturing a plethora of unique and distinct cultures and belief systems. Our sample size did not allow us to break the sample down into various countries of origin (e.g., Japanese-American, Chinese-American), and the vast majority of our sample were Chinese-American, but we acknowledge that in using the broad category of "AsianAmerican" we may have overlooked some important cultural differences that may impact the parent-child relationship during emerging adulthood. Future work should not only examine more closely the potential differences that exist within Asian-American communities but also within other ethnic and religious subcultures in the United States, and across other cultures and countries around the world.

A third limitation is that our analyses used only two waves of a data. As noted previously, there was a high level of stability not only in prosocial behavior from Time 1 to Time 2 but in most of the parenting variables as well so employing only two points in time might not provide a wide enough window to see all of the ways in which young people and parents are affecting one another with their behaviors. Future work should employ more waves of data across a longer period of time in the third decade of life. In doing so, future work should also consider other possible moderators when examining the relations between prosocial behavior and parenting including child characteristics, parent characteristics, and aspects of the overall parent-child relationship.

Despite the limitations, this study makes several important contributions to understanding the dynamic process of 
human development in which children both influence and are influenced by their parents. Indeed, current findings support the bidirectional relations between parenting and the emerging adults' prosocial behavior toward family members, with particular emphasis placed on the role of the child. Moreover, the findings in regard to ethnicity underscore the need for researchers to take into account sub-group differences when studying aspects of the parent-child relationship, perhaps especially when considering the role of the father. The current study highlights the need for more dynamic and culturally sensitive research to more accurately capture the multifaceted nature of prosocial development during the third decade of life.

Acknowledgements The authors express appreciation to the instructors and students at all Project READY data collection sites for their assistance. We also are grateful for the grant support of the Family Studies Center and the School of Family Life at Brigham Young University.

\section{References}

Aquilino, W. S. (1997). From adolescent to young adult: A prospective study of parent-child relations during the transition to adulthood. Journal of Marriage and Family, 59(3), 670-686.

Aquilino, W. S. (2006). Family relationships and support systems in emerging adulthood. In J. J. Arnett \& J. L. Tanner (Eds.), Emerging adults in America: Coming of age in the 21st century (pp. 193-217). Washington, DC: American Psychological Association. doi: 10.1037/11381-008.

Arbuckle, J. L. (2010). Amos 19.0 user's guide. Chicago, IL: SPSS, Amos Development Corporation.

Arnett, J. J. (1998). Learning to stand alone: The contemporary American transition to adulthood in cultural and historical context. Human Development, 41, 295-315.

Arnett, J. J. (2000). Emerging adulthood: A theory of development from the late teens through the twenties. American Psychologist, 55(5), 469. doi: 10.1037/0003-066X.55.5.469.

Arnett, J. J. (2014). Emerging adulthood: The winding road from the late teens through the twenties. NY: Oxford Press.

Barber, B. K. (1996). Parental psychological control: Revisiting a neglected construct. Child Development, 67, 3296-3319.

Barry, C. M., Padilla-Walker, L. M., Madsen, S. D., \& Nelson, L. J. (2008). The impact of maternal relationship quality on emerging adults' prosocial tendencies: Indirect effects via regulation of prosocial values. Journal of Youth and Adolescence, 37, 581-591. doi: 10.1007/s10964-007-9238-7.

Bronfenbrenner, U., \& Morris, P. A. (2006). The bioecological model of human development. In R. M. Lerner \& W. Damon (Eds.), Handbook of child psychology (Vol 1, 6th edn., pp. 793-828). Hoboken, NJ: Wiley \& Sons.

Carlo, G., McGinley, M., Hayes, R., \& Martinez, M. (2012). Empathy as a mediator of the relations between parent and peer attachment and prosocial and physically aggressive behaviors in Mexican American college students. Journal of Social and Personal Relationships, 29, 337-357.

Chao, J. (2001). Asian American philanthropy: Acculturation and charitable vehicles. In P. Rodgers (Ed.), Philanthropy in communities of color: Traditions and challenges (Vol. 1, pp. 57-80).,
No. 1). Indianapolis, IN: Association for Research on Nonprofit Organizations and Voluntary Action.

Chao, R. K. (1994). Beyond parental control and authoritarian parenting style: Understanding Chinese parenting through the cultural notion of training. Child Development, 65(4), 1111-1119.

Chao, R. K. (2000). The parenting of immigrant Chinese and European American mothers: Relations between parenting styles, socialization goals, and parental practices. Journal of Applied Developmental Psychology, 21(2), 233-248.

Chen, X., Liu, M., Rubin, K. H., Cen, G.-z., Gao, X., \& Li, D. (2002). Sociability and prosocial o orientation as predictors of youth adjustment: A seven-year longitudinal study in a Chinese sample. International Journal of Behavioral Development, 26(2), $128-136$.

Coley, R., Votruba-Drzal, E., \& Schindler, H. (2009). Fathers' and mothers' parenting predicting and responding to adolescent sexual risk behaviors. Child Development, 80, 808-827.

Dekovic, M., \& Janssens, J. M. (1992). Parents' child-rearing style and child's sociometric status. Developmental Psychology, 28, 925-932.

Eberly, M. B., \& Montemayor, R. (1998). Doing good deeds: An examination of adolescent prosocial behavior in the context of parent/adolescent relationships. Journal of Adolescent Research, $13,403-432$.

Eisenberg, N., Fabes, R. A., Shepard, S. A., Guthrie, I. K., Murphy, B. C., \& Reiser, M. (1999). Parental reactions to children's negative emotions: longitudinal relations to quality of children's social functioning. Child Development, 70(2), 513-534.

Eisenberg, N., Fabes, R. A., \& Spinrad, T. L. (2006). Prosocial development. In N. Eisenberg, W. Damon \& R. M. Lerner (Eds.), Handbook of child psychology: Vol. 3. Social, emotional and personality development (6th edn., pp. 646-718). New York: Wiley.

Eisenberg, N., Guthrie, I. K., Cumberland, A., Murphy, B. C., Shepard, S. A., Zhou, Q., \& Carlo, G. (2002). Prosocial development in early adulthood: A longitudinal study. Journal of Personality and Social Psychology, 82, 993-1006.

Eisenberg, N., Wolchik, S. A., Goldberg, L., \& Engel, I. (1992). Parental values, reinforcement, and young children's prosocial behavior: A longitudinal study. Journal of Genetic Psychology, 153(1), 19-36.

Fish, M., \& Crockenberg, S. (1986). Correlates and antecedents of nine-month infant behavior and mother-infant interaction. Infant Behavior and Development, 4, 69-81.

Fu, G., Lee, K., Cameron, C. A., \& Xu, F. (2001). Chinese and Canadian adults' categorization and evaluation of lie-and truth-telling about prosocial and antisocial behaviors. Journal of Cross-Cultural Psychology, 32(6), 720-727.

Grolnick, W. S., Ryan, R. M., \& Deci, E. L. (1991). The inner resources for school performance: Motivational mediators of children's perceptions of their parents. Journal of Educational Psychology, 83, 508-517.

Hastings, P. D., Zahn-Waxler, C., Robinson, J., Usher, B., \& Bridges, D. (2000). The development of concern for others in children with behavior problems. Developmental Psychology, 36, 531-546.

Ho, D. Y. F. (1986). Chinese pattern of socialization: A critical review. In M. H. Bond (Ed.), The psychology of the Chinese people (pp. 1-37). New York, NY: Oxford University Press.

Ho, E. S. C., \& Willms, J. D. (1996). The effects of parental involvement on eighth grade achievement. Sociology Education, 69, $126-141$.

Hwang, K. K. (1999). Filial piety and loyalty: Two types of social identification in Confucianism. Asian Journal of Social Psychology, 2(1), 163-183. doi: 10.1111/1467-839X.00031.

Jin, P., Sun, G., \& Chen, Q. (2013). Study on the relationship between prosocial behavior and adaptation of college students. Chinese Journal of Economic Herald, 5, 76-77. 
Kerr, M., \& Stattin, H. (2000). What parents know, how they know it, and several forms of adolescent adjustment: Further support for a reinterpretation of monitoring. Developmental Psychology, 36, 366-380.

Kou, Y., Ma, Y., \& Tang, C. (2004). Chinese College Students' Prosocial Tendencies, Prosocial Moral Reasoning and Their Correlation Patterns. Chinese Journal of Psychological Science, 27(2), 329-332.

Krevans, J., \& Gibbs, J. C. (1996). Parents' use of inductive discipline: Relations to children's empathy and prosocial behavior. Child Development, 67(6), 3263-3277.

Laible, D. J., Carlo, G., \& Roesch, S. C. (2004). Pathways to selfesteem: The role of parent and peer attachment, empathy, and social behaviors. Journal of Adolescence, 27, 703-716.

Lam, W. A., \& McCullough, L. B. (2000). Influence of religious and spiritual values on the willingness of Chinese-Americans to donate organs for transplantation. Clinical transplantation, 14(5), $449-456$.

LeMoyne, T., \& Buchanan, T. (2011). Does "hovering" matter? Helicopter parenting and its effect on well-being. Sociological Spectrum, 31, 399-418.

Li, Z., Yin, X., Cai, T., \& Su, L. (2012). Role of father-child attachment on the relation between father involvement and children's prosocial behavior. Chinese Journal of Clinical Psychology, 20(5), 705-707.

Little, T. D. (2013). Longitudinal structural equation modeling. New York: Guilford.

Luyckx, K., Soenens, B., Vansteenkiste, M., Goossens, L., \& Berzonsky, M. (2007). Parental psychological control and dimensions of identity formation in emerging adulthood. Journal of Family Psychology, 21, 546-550.

Ma, H. K. (1989). Moral orientation and moral judgment in adolescents in Hong Kong, Mainland China, and England. Journal of CrossCultural Psychology, 20, 152-177.

Masten, A. S., Burt, K. B., Roisman, G. I., Obradovic, J., Long, J. D., \& Tellegen, A. (2004). Resources and resilience in the transition to adulthood: Continuity and change. Development \& Psychopathology, 16, 1071-1094.

Moilanen, K., Rasmussen, K., \& Padilla-Walker, L. M. (2015). Bidirectional associations between self-regulation and parenting styles in early adolescence. Journal of Research on Adolescence, 25, $246-262$.

Nelson, L. J., \& Padilla-Walker, L. M. (2013). Flourishing and floundering in emerging-adult college students. Emerging Adulthood, $1,67-78$

Nelson, L. J., Padilla-Walker, L. M., Carroll, J. S., Madsen, S. D., Barry, C. M., \& Badger, S. (2007). "If you want me to treat you like an adult, start acting like one!" Comparing the criteria that emerging adults and their parents have for adulthood. Journal of Family Psychology, 21(4), 665.

Nelson, L. J., Padilla-Walker, L. M., Christensen, K. J., Evans, C. A., \& Carroll, J. A. (2011). Parenting in emerging adulthood: An examination of parenting clusters and correlates. Journal of Youth and Adolescence, 40, 730-743.

Newton, E., Laible, D., Carlo, G., Steele, J., \& McGinley, M. (2014). Do sensitive parents foster kind children, or vice versa? Bidirectional influences between children's prosocial behavior and parental sensitivity. Developmental Psychology, 50, 1808-1816.

Padilla-Walker, L., Nelson, L., Madsen, S., \& Barry, C. (2008). The role of perceived parental knowledge on emerging adults' risk behaviors. Journal of Youth and Adolescence, 37(7), 87-859.

Padilla-Walker, L. M. (2015). Moral development during emerging adulthood. In J. J. Arnett (Ed.), The Oxford handbook of emerging adulthood (pp. 449-463). New York, NY: Oxford University Press.
Padilla-Walker, L. M., Carlo, G., Christensen, K. J., \& Yorgason, J. B. (2012). Bidirectional relations between authoritative parenting and adolescents' prosocial behaviors. Journal of Research on Adolescence, 22(3), 400-408.

Padilla-Walker, L. M., Carlo, G., \& Nielson, M. G. (2015). Does helping keep teens protected? Longitudinal bidirectional relations between prosocial behavior and problem behavior. Child Development, 86, 1759-1772.

Padilla-Walker, L. M., \& Christensen, K. J. (2011). Empathy and selfregulation as mediators between parenting and adolescents' prosocial behaviors toward strangers, friends, and family. Journal of Research on Adolescence, 21, 545-551.

Padilla-Walker, L. M., Nielson, M. G., \& Day, R. A. (2014). Positive and negative parenting and adolescents' prosocial behavior toward three targets. Unpublished manuscript..

Perez-Albeniz, A. A., \& de Paul, J. (2004). Gender differences in empathy in parents at high- and low-risk of child physical abuse. Child Abuse and Neglect, 28(3), 289-300.

Peterson, C., \& Seligman, M. E. P. (2004). Character strengths and virtues: A handbook and classification. Washington, DC: Oxford University Press.

Qin, D. B. (2006). Our child doesn't talk to us anymore: Alienation in immigrant Chinese families. Anthropology \& Education Quarterly, 37(2), 162-179.

Qin, D. B. (2008). Doing well vs. feeling well: Understanding family dynamics and the psychological adjustment of Chinese immigrant adolescents. Journal of Youth and Adolescence, 37(1), 22-35.

Qin, D. B., Way, N., \& Mukherjee, P. (2008). The other side of the model minority story the familial and peer challenges faced by Chinese American adolescents. Youth \& Society, 39(4), 480-506.

Randall, B. A., \& Wenner, J. R. (2014). Adopting a multidimensional perspective on college students' prosocial behaviors. In L. M. Padilla-Walker \& G. Carlo (Eds.), Prosocial development: A multidimensional perspective (pp. 374-392). New York, NY: Oxford University Press.

Robbins, R. J. (1994). An assessment of perceptions of parental autonomy support and control: Child and parent correlates. Unpublished Doctoral Dissertation.

Roth, G. (2008). Perceived parental conditional regard and autonomy support as predictors of young adults' self- versus other-oriented prosocial tendencies. Journal of Personality, 76, 513-533.

Rubin, K. H., Nelson, L. J., Hastings, P., \& Asendorpf, J. (1999). The transaction between parents' perceptions of their children's shyness and their parenting styles. International Journal of Behavioral Development, 23, 937-957.

Smits, I., Soenens, B., Luyckx, K., Duriez, B., Berzonsky, M., \& Goossens, L. (2008). Perceived parenting dimensions and identity styles: Exploring the socialization of adolescents' processing of identity-relevant information. Journal of Adolescence, 31(2), 151-164.

Soucie, K., Lawford,H. L., \& Pratt, M. W. (2012). Personal stories of empathy in adolescence and emerging adulthood. Merrill Palmer Quarterly, 58, 141-158.

Steinberg, L., Dornbusch, S., \& Brown, B. (1992). Ethnic differences in adolescent achievement: An ecological perspective. American Psychologist, 47, 723-729.

Urry, S., Nelson, L. J., \& Padilla-Walker, L. M. (2011). Mother knows best: Correlates of child disclosure and maternal knowledge in emerging adulthood. Journal of Family Studies, 17, 157-173.

Wang, L., Chen, X., Chen, H., Cui, L., \& Li, M. (2006). Affect and maternal parenting as predictors of adaptive and maladaptive behaviors in Chinese children. International Journal of Behavioral Development, 30(2), 158-166.

Wartman, K., \& Savage, M. (2008). Parental involvement in higher education: Understanding the relationship among students, 
parents, and the institution. ASHE Higher Education Report, 33(6), 1-125. San Francisco, CA: Jossey-Bass.

Willoughby, B. J., Hersh, J., Padilla-Walker, L. M., \& Nelson, L. J. (2015). "Back off!": Helicopter parenting and a retreat from marriage among emerging adults. Journal of Family Issues, 36, $669-692$.

$\mathrm{Wu}, \mathrm{X}$. (2009). The relationship between self-esteem, parenting style, and prosocial behavior of college students. Unpublished Master Thesis, Shaanxi Normal University.
Xu, Y., Farver, J. A. M., Zhang, Z., Zeng, Q., Yu, L., \& Cai, B. (2005). Mainland Chinese parenting styles and parent-child interaction. International Journal of Behavioral Development, 29(6), 524-531.

Yao, X. (2000). An introduction to Confucianism. New York, NY: Cambridge University Press. 